\title{
Multidisciplinary Intention: Revealing The Consequence of Psychosocial Factors on the Adherence to Treatment of the Patients with Type 2 Diabetes Mellitus
}

\author{
Anna Kohut1, O. Potapov², I. Frankova ${ }^{3}$, M. Bobryk ${ }^{3}$, J. Komisarenko ${ }^{3}$, O. Chaban ${ }^{3}$ \\ ${ }^{1}$ Communal Institution of Kyiv Regional Council "Regional Psychiatric and Narcological \\ Medical Association”, Glevakha, Ukraine \\ 2 Center for Innovative Medical Technologies of the National Academy of Sciences of \\ Ukraine, Kyiv, Ukraine \\ 3 Bogomolets National Medical University, Kyiv, Ukraine
}

\begin{abstract}
.
Background: Diabetes Mellitus type 2 (T2DM) has been becoming a global world health problem affecting countries with different income level (Mogre, Johnson, Tzelepis, Shaw, \& Paul, 2017). The low level of compliance and the increased level of the diabetes distress has been closely associated with destitute glycemic control. Some diabetes-specific psychological variables may be important for psychological status of patients and glycemic control to the achievement of individual glycemic targets (Indelicato et al., 2017). Therefore, alexithymia - psychological clinical characteristic of the reduced emotional component that could have been associated with the metabolic syndrome in patients with type 2 diabetes, for example (Lemche, Chaban, \& Lemche, 2014). It is important to consider the clinical evaluation of mental aspects in patients with T2DM, however, in order to preventing potentially unfavorable self-care behavior leading to complications of this disease, including a decrease in the quality of life level (Conti et al., 2017). Patient's level of the quality of life is the one of main important components of treatment adherence and clinical decision making for improving the effectiveness of therapy (Chaban, Khaustova, \& Bezsheiko, n.d.). The low level of medication compliance and quality of life could have been addicted in consequence to non-adherence and respectively - inefficiency of therapy of these patients. Therefore, multidisciplinary connection - involving mental health care specialists can potentially improve treatment effectiveness (Kogut at al., 2018).
\end{abstract}

Purpose: Aim of the study was-comprehensive assessment of psychological status and quality of life of the patients with T2DM to explore the relationships with their commitment to treatment depending by the level of compliance for medication.

Method: Study population ( $\mathrm{n}=40$ ) consists of the patients with T2DM (male -32 , $5 \%$, female $-67,5 \%$ ), married $-60 \%$, widow $-25 \%$, single $-15 \%$; had higher completed education $-60 \%$, had the different level of obesity $-50 \%$ of patients. 
Study data have been collected with the demographic data (including: age, BMI, the level of arterial pressure, blood glucose and glycated hemoglobin (HbA1c) laboratory results); Chaban Quality of Life Scale (CQLS), Medication Compliance Scale (MCS), Holmes and Rahe Stress Scale (HRSS), Dysfunctional attitudes Scale (DAS), Toronto Alexithymia Scale (TAS-20) and the Depression, Anxiety and Stress Scale (DASS-21)). Glycemic control was based on HbA1c results. The statistics analysis has been performed by using descriptive statistics and Pearson's correlation with SPSS Statistics 23.0.

Results: Statistically significant difference has been found between the groups of the patients with low, middle and high level of their compliance to therapy according to MCS results. Strong relationships have been found between the numeral scores by MCS with the DAS ( $<<0,0001 ; r=-0,57)$, TAS-20 ( $p=0,006 ; r=$ $-0,42)$, HRSS $(p=0,001 ; r=-0,524)$ and DASS-21 (depression $(p<0,0001 ; r=$ $-0,703)$, anxiety $(\mathrm{p}=0,001 ; \mathrm{r}=-0,498)$, stress $(\mathrm{p}<0,0001 ; \mathrm{r}=-0,62))$ results significant bilateral indirect correlation and direct by CQLS results of scores on level $(p=0,22 ; r=0,361)$. Therefore, statistical difference have been detected between the results of: glucose level $(p=0,032 ; r=-0,349)$; depending by the belonging to the group of different results between level of cognitive distortions (DAS) $(\mathrm{p}=0,001 ; \mathrm{r}=-0,508)$, alexythimia (TAS-20) $(\mathrm{p}=0,001 ; \mathrm{r}=-0,498)$, stress $(\mathrm{p}<0,0001 ; \mathrm{r}=-0,67)$, depression $(\mathrm{p}<0,0001 ; \mathrm{r}=-0,71)$, anxiety $(\mathrm{p}=0,007 ; \mathrm{r}=$ $-0,418)(\mathrm{DASS}-21)$, stress resistance (HRSS) $(\mathrm{p}=0,008 ; \mathrm{r}=-0,416)$ and quality of life $(\mathrm{p}=0,44 ; \mathrm{r}=0,32)$.

Based on the results of research - there has been found the strong relationship between the results of MCS with low level of compliance and high level by the scores of psychometric scales, as evidenced, that these patients would be have low rates of treatment effectiveness in the future.

Conclusions: The possible psychosocial factors affecting the level of compliance and psychological status indicators in patients with Diabetes Mellitus type 2, which have been determined based on the results of research, such as the level of: quality of life, stress resistance, alexithymia, cognitive distortions, anxiety, depression and distress of these patients. Therefore, patients with a low level of commitment to treatment have had a higher risk of treatment failure and needed qualified help from specialists on various failures to ensure the multidisciplinary approach to the treatment of patients with Diabetes Mellitus type 2.

Originality: The concept of "adherence to treatment" is so comprehensive, in contrast to the concept of "compliance" or "commitment" to treatment, in a medication aspect.

Limitations of the study: The low sample of T2DM patients, that has been planning to correction in further researches.

Strengths of the study: The urgency of this problem in the vast field of research 
and development in both medical and social sciences. The multidisciplinary approach would have been promising and innovative direction for the future.

Key words: diabetes, health, health care, medical, medical research, medical science, medicine, mental health, mental stress, psychology, quality of life, social well-being

References

1. Chaban, O., Khaustova, O., \& Bezsheiko, V. (n.d.). Reliability and validity of Chaban Quality of Life Scale. Retrieved from https://www.ecnp.eu/presentationpdfs/70/P.2.h.301.pdf

2. Conti, C., Di Francesco, G., Fontanella, L., Carrozzino, D., Patierno, C., Vitacolonna, E., \& Fulcheri, M. (2017). Negative Affectivity Predicts Lower Quality of Life and Metabolic Control in Type 2 Diabetes Patients: A Structural Equation Modeling Approach. Front Psychol. 24;8:831. doi: 10.3389/fpsyg.2017.00831

3. Mogre, V., Johnson, N., Tzelepis, F., Shaw, J., \& Paul, C. (2017). Adherence to self-care behaviours and associated barriers in type 2 diabetes patients of low-and middle-income countries: a systematic review protocol. Syst Rev. 2017 Feb 27;6(1):39. doi: 10.1186/s13643-017-0436-4.

4. Indelicato, L., Dauriz, M., Santi, L., Bonora, F., Negri, C., Cacciatori, V., Targher, G., Trento, M., \& Bonora, E. (2017). Psychological distress, self-efficacy and glycemic control in type 2 diabetes. Metab Cardiovasc Dis. 2017 Apr;27(4):300-306. doi: 10.1016/j.numecd.2017.01.006. Epub 2017 Jan 31.

5. Lemche, A., Chaban, O., \& Lemche, E. (2014). Alexithymia as a risk factor for type 2 diabetes mellitus in the metabolic syndrome: a cross-sectional study. Psychiatry Res. 215(2):438-43. doi: 10.1016/j.psychres.2013.12.004

6. Kogut, A., Bobryk, M., Potapov, O., Frankova, I., Komisarenko, J., Khaustova, O., \& Chaban, O. (2018). Multidisciplinary Approach to Research of the Adherence to Treatment Phenomenon in Patients with Diabetes Mellitus. Mental Health: Global Challenges Journal, Rome (pp. 40-41). DOI: http://doi.org/10.32437/MHGCJ-2018 